\title{
Unravelling effects of relative humidity on lipid barrier formation in human skin equivalents
}

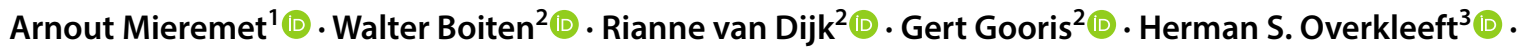 \\ Johannes M. F. G. Aerts ${ }^{4}\left[\right.$. Joke A. Bouwstra ${ }^{2} \mathbb{D}$. Abdoelwaheb El Ghalbzouri ${ }^{1} \mathbb{D}$
}

Received: 18 January 2019 / Revised: 19 June 2019 / Accepted: 2 July 2019 / Published online: 18 July 2019

(c) The Author(s) 2019

\begin{abstract}
Relative humidity (RH) levels vary continuously in vivo, although during in vitro generation of three-dimensional human skin equivalents (HSEs) these remain high (90-95\%) to prevent evaporation of the cell-culture medium. However, skin functionality is directly influenced by environmental RH. As the barrier formation in HSEs is different, there is a need to better understand the role of cell-culture conditions during the generation of HSEs. In this study, we aim to investigate the effects of RH on epidermal morphogenesis and lipid barrier formation in HSEs. Therefore, two types of HSEs were developed at $90 \%$ or at $60 \% \mathrm{RH}$. Assessments were performed to determine epidermal morphogenesis by immunohistochemical analyses, ceramide composition by lipidomic analysis, and lipid organization by Fourier transform infrared spectroscopy and smallangle X-ray diffraction. We show that reduction of RH mainly affected the uppermost viable epidermal layers in the HSEs, including an enlargement of the granular cells and induction of epidermal cell activation. Neither the composition nor the organization of the lipids in the intercorneocyte space were substantially altered at reduced $\mathrm{RH}$. In addition, lipid processing from glucosylceramides to ceramides was not affected by reduced RH in HSEs as shown by enzyme expression, enzyme activity, and substrate-to-product ratio. Our results demonstrate that RH directly influences epidermal morphogenesis, albeit the in vitro lipid barrier formation is comparable at $90 \%$ and $60 \% \mathrm{RH}$.
\end{abstract}

Keywords Artificial skin $\cdot$ Humidity $\cdot$ Cell-culture techniques $\cdot$ Ceramides $\cdot$ Molecular probes

Joke A. Bouwstra and Abdoelwaheb El Ghalbzouri Joint senior authorship.

Electronic supplementary material The online version of this article (https://doi.org/10.1007/s00403-019-01948-3) contains supplementary material, which is available to authorized users.

Abdoelwaheb El Ghalbzouri

A.Ghalbzouri@lumc.nl

1 Department of Dermatology, Leiden University Medical Center, Albinusdreef 2, Room C3-76, 2333 ZA Leiden, The Netherlands

2 Research Division BioTherapeutics, Leiden Academic Centre for Drug Research, Leiden University, Leiden, The Netherlands

3 Department of Bio-organic Synthesis, Leiden Institute of Chemistry, Leiden University, Leiden, The Netherlands

4 Medical Biochemistry, Leiden Institute of Chemistry, Leiden University, Leiden, The Netherlands

\section{Introduction}

Main functions of the skin are to protect the body from water loss and against exogenous factors, which are primarily performed by the stratum corneum (SC). Skin functionality is influenced by environmental relative humidity $(\mathrm{RH})$, which ranges worldwide from 5-99\% RH with a median of 70-80\% $\mathrm{RH}$ over land areas [10]. The RH affects susceptibility to mechanical stress and fracture, elasticity, protein organization, lipid conformation, and epidermal hydration $[4,8,15$, $18,34,42,55]$. In vivo studies revealed that high $\mathrm{RH}$ levels delayed epidermal barrier repair and reduced the level of natural moisturizing factor $[15,18]$. Furthermore, switching from high to low RH affected total epidermal water loss (TEWL) values, a widely applied analysis to determine the skin barrier function [37, 23, 26]. This indicates the adaptation of the skin barrier formation to fluctuating RH levels [45].

Despite the interactions between $\mathrm{RH}$ and the skin, during the generation of three-dimensional human skin equivalents 
(HSEs), high RH levels (90-95\%) are used as the standard cell-culture condition. This difference between in vivo and in vitro RH levels could contribute to the altered barrier formation of HSEs compared to that of native human skin (NHS) $[13,36,38,48]$. Only a limited amount of comparative studies addressed the influence of RH levels in vitro [6, $7,28,46,47]$. These showed that epidermal morphogenesis was affected by a reduction in RH, albeit not uniformly in all HSE types. Moreover, an improved skin hydration was observed, at which $60 \%$ RH was reported to be an optimal in vitro level [6]. Furthermore, the HSEs developed at reduced RH were shown to have enhanced barrier properties [46]. Nevertheless, it remains unresolved by which mechanism the epidermal barrier is reinforced.

The SC consists of fully keratinized corneocytes surrounded by a lipid matrix, which is the only continuous pathway through the SC $[14,40]$. Therefore, the lipid matrix is highly important for the functionality of the epidermal barrier. It consists of a specific lipid composition (i.e., ceramides, cholesterol, and free fatty acids), assembled in a characteristic lipid organization (i.e., lateral and lamellar). The 12 most common ceramide subclasses were analysed in this study, referred to as total ceramides $\left(\mathrm{CERs}_{\text {total }}\right)$. These were subcategorized in $\omega$-esterified ceramide (CERs EO) and in (non- $\omega$-esterified) ceramide (CERs) subclasses [25, $53,54]$. The previous studies revealed a different composi-

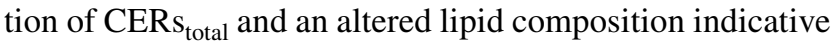
for the altered barrier formation in HSEs as compared to NHS $[48,50]$.

The interaction between $\mathrm{RH}$ and the lipid barrier formation in HSEs has been studied in submerged $(>100 \% \mathrm{RH})$ and in reduced $\mathrm{RH}(<90 \% \mathrm{RH})$ conditions. When developed at submerged conditions ( $>100 \% \mathrm{RH})$, the level of ceramide precursor (acyl)glucosylceramide was elevated, leading to a lower level of ceramide EOS as compared to air-exposed cultures (93\%) [49]. This could be a consequence of elevated $\mathrm{pH}$ values at submerged or occluded conditions [42], which may affect the hydrolytic activities of $\beta$-glucocerebrosidase-1 (GBA) or acid sphingomyelinase (aSMASE). These enzymes convert glucosylceramide and sphingomyelin, respectively, to ceramide at the stratum granulosum (SG)-SC interface [16, 52]. Opposite to submerged conditions, in HSEs generated at reduced RH (50\%), the expression of glucosylceramide synthase was upregulated [46], indicating that RH is associated with biosynthesis and processing of glucosylceramides. Nevertheless, it remains to be determined whether RH levels directly affect lipid composition and organization in the SC of in vitro developed HSEs.

In this study, we aim to determine if RH levels affect epidermal morphogenesis and barrier lipid formation in HSEs. Therefore, full-thickness models (FTMs) and collagen-chitosan full-thickness models (CC-FTMs) were generated at
$90 \%$ and $60 \%$ RH. Both HSE types encompass the epidermal and dermal equivalents, although the CC-FTM has a strengthened dermal extracellular matrix due to the added biopolymer chitosan and improved barrier formation [30]. In addition, we examined the conversion of precursor glucosylceramide to ceramide at the SG-SC interface to obtain more detailed information on this process at various $\mathrm{RH}$ levels.

\section{Materials and methods}

\section{Generation of FTMs and CC-FTM at reduced RH}

Declaration of Helsinki principles is followed during the obtainment of primary cells from surplus female adult mama skin tissue, as stated earlier [21, 29]. Isolation of primary cells from the dermis and epidermis and cell culturing was performed as described before [12, 51]. All isolated primary cells were tested and found negative for mycoplasma contamination. FTMs and CC-FTMs were generated as described before $[30,39,48]$. The reduction in $\mathrm{RH}$ was performed from $90 \%$ gradually in steps of $7.5 \%$ each day initiated after 7 day air exposure to $60 \%$ using a Memmert INC153med $\mathrm{CO}_{2}$ incubator with humidity module (Memmert, Schwabach, Germany). Medium was refreshed every other day after 7 day air exposure. HSEs were developed for a total of 16 day air-exposed. HSE batches were generated with four unique primary cell donors.

\section{Immunohistochemical analyses}

Sections of HSEs or NHS were formaldehyde fixated and embedded in paraffin or snap frozen in liquid nitrogen [31]. Haematoxylin and eosin (HE) staining was performed according to manufacturer's instructions (Klinipath, Duiven, The Netherlands). Protein analyses by immunohistochemistry or indirect immunofluorescence were performed on $5 \mu \mathrm{m}$ sliced paraffin embedded cross sections. After deparaffinization and rehydration, heat-mediated antigen retrieval in citrate buffer $\mathrm{pH} 6$ occurred. Antigen retrieval for collagen type IV staining was mediated by protease incubation. Nonspecific antibody binding was reduced through blocking with normal human serum (Sanquin, Leiden, The Netherlands). Immunohistochemical analyses were performed using the streptavidin-biotin-peroxidase system (GE Healthcare, Buckinghamshire, United Kingdom) according to the manufacturer's instructions or using indirect immunofluorescence, as described before [30]. Specifications of the antibodies are provided in supplementary Table 1. Control immunostainings revealed no unspecific binding or background staining of the secondary antibody (Supplementary Fig. S1a). Number of corneocyte layers was determined by safranin red staining and potassium hydroxide expansion of the SC 
$[43,44]$. Estimations of the epidermal thickness and the proliferation index were performed as reported earlier [1, 30]. Activity-based probe assay using MDW941 was performed as reported earlier by van Smeden et al. [52].

\section{Stratum corneum lipid composition}

Extraction of SC lipids was performed based on an adjusted Bligh and Dyer method as described by Boiten et al. [2]. The dry SC was weighed before and after lipid extraction using a microbalance. The lipids were analysed by liquid chromatography coupled to mass spectrometry (LC-MS) according to the methods as described elsewhere [2]. Details of instrument settings and data quantification are described in supplementary materials and methods.

\section{Stratum corneum lipid organization}

Determination of lamellar organization was performed by small-angle X-ray diffraction [19] and determination of lateral organization was performed by Fourier transform infrared spectroscopy (FTIR) [32,33], according to methods described in supplementary materials and methods.

\section{Statistics}

Statistical analyses are conducted using GraphPad Prism version 7.00 for Windows (GraphPad Software, La Jolla California USA). Statistical testing was performed with one-way or two-way ANOVA with Tukey's post-test. Significance is shown for comparison between high and low RH (with lines) within an HSE type and for comparison between all HSEs versus NHS (above NHS), otherwise specifically stated. No comparisons between FTMs and CC-FTMs were performed due to primary donor effect. Statistical differences are noted as *, ** or ***, corresponding to $P<0.05$, $<0.01,<0.001$.

\section{Results}

\section{Epidermal morphogenesis in HSEs developed at reduced relative humidity}

To thoroughly and robustly assess the effect of RH in HSEs, we generated FTMs and CC-FTMs at $90 \%$ and $60 \%$ RH. The reduction in $\mathrm{RH}$ was performed gradually after the first SC layers were formed to protect the viable epidermis from excessive desiccation [6]. Assessments of macroscopic appearance and general morphology were performed for CCFTMs and NHS (Fig. 1a) and for FTMs (Supplementary Fig. S2a). The epidermis contained four distinguishable epidermal layers in both HSE types, irrespective of RH levels. In addition, the thickness of the viable epidermis and number of corneocyte cell layers in the SC was similar at $90 \%$ and $60 \%$ RH in both HSE types (Fig. 1b, c). In HSEs generated for 16 days, the number of corneocyte layers was slightly higher than that of NHS. Noteworthy, the corneocytes of both HSE types expanded more in the alkali environment that the corneocytes of NHS (Supplementary Fig. S1b).

The expression of protein biomarkers in HSEs and NHS was evaluated to assess the effect of RH on epidermal morphogenesis, basement membrane (BM) formation, and fibroblast distribution in CC-FTMs and NHS (Fig. 1d), and in FTMs (Supplementary Fig. S2b). The epidermal terminal and late differentiation programs were examined by expression of loricrin, filaggrin, and involucrin. This revealed an increased thickness of the SG in CC-FTMs generated at $60 \%$ RH, but not in FTMs at 60\% RH. Involucrin was also expressed in the spinous layer of HSEs, in contrast to its restricted expression in the SG of NHS. Similar execution of the early differentiation program was detected in all conditions based on the suprabasal keratin 10 expression. Epidermal activation was increased at reduced RH in CC-FTMs based on the expression of keratin 16 (Fig. 1d). Elevated epidermal activation could not be detected in FTMs, due to the expression of keratin 16 at 90\% RH. In all HSEs and NHS, the expression of keratin 17 was absent, indicating no severe epidermal activation induced by reduced RH (Supplementary Fig. S2c). The cell proliferation marker Ki67 remained located at the basal layer in all conditions, with comparable proliferation indexes at high and low RH. The expression of collagen type IV was unaffected by the reduced RH, indicating similar BM formation. Furthermore, the distribution of the fibroblasts was analysed by vimentin expression, which was similar at both RH levels. As compared to NHS, the deposition of the BM was reduced and the distribution of the fibroblasts was homogeneous. Altogether, these results indicate that reduction in RH mainly affected the uppermost layers of the epidermis.

\section{Lipidomic profiling of stratum corneum ceramides}

To gain more information on the barrier formation, the ceramide composition was investigated using an advanced liquid chromatography coupled to mass spectrometry (LC-MS)

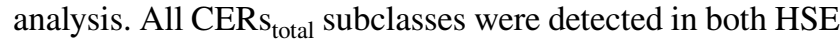
types and in NHS on the LC-MS ion maps (Fig. 2a). An increased presence of low mass CERs was detected (below 600 atomic mass units) in HSEs compared to NHS. Quantification of the detected signal was performed to determine the level of CERs total $_{\text {p }}$ per $\mathrm{mg}$ SC. This revealed no significant change in the absolute amount of CERs $\mathrm{stal}_{\text {tol }}$ after generating HSEs at reduced RH compared to high RH (Fig. 2b). In addition, a comparison between the cumulative detected $\mathrm{CERs}_{\text {total }}$ per injection in the LC-MS revealed similarity in 
Fig. 1 Generation of HSEs at reduced relative humidity. a General morphology of CC-FTMs generated at high $(90 \%)$ and low $(60 \%)$ $\mathrm{RH}$, assessed by eye or after HE staining. b Epidermal thickness of HSEs and of NHS, indicated by mean $+\mathrm{SD}, N=4$. c Number of corneocyte layers in HSEs and in NHS. Data indicates mean $+\mathrm{SD}, N=4$. d Epidermal morphogenesis, basement membrane formation, and fibroblast distribution in CC-FTMs and NHS. Expression of loricrin (LOR), filaggrin (FLG), involucrin (INV), keratin 10 (K10), and keratin 16 (K16) indicates epidermal differentiation programs or activation. Proliferation was assessed by Ki67 (with indicated proliferation index as mean $\pm \mathrm{SD}, N=4$ ), basement membrane formation by collagen type IV (COL IV), and fibroblast distribution by vimentin (VIM) expression. Protein biomarkers are shown in red and nuclei in blue. Yellow dotted line indicates dermal-epidermal junction. Scale bar indicates $100 \mu \mathrm{m}$

HSEs generated at high and low RH (Fig. 2c). The mean carbon chain length (MCL) of CERs and CERs EO was calculated, which revealed no significant difference for neither the MCL of CERs nor for the MCL of CERs EO between high and low RH (Fig. 2d, e). The MCL of CERs in NHS was significantly higher as compared to HSEs, whereas the MCL of CERs EO in NHS was more comparable to that of HSEs. More detailed information on the chain length of CERs and CERs EO was obtained by plotting the distribution by carbon atom number, for even numbered CERs ranging from C32 (32 carbon atoms) to C54 and for CERs EO ranging from C64 to C74 (Supplementary Fig. S3a-d). This revealed no substantial differences between high and low $\mathrm{RH}$ levels, solely CER EO C68 in CC-FTMs was significantly reduced at $60 \% \mathrm{RH}$. Compared to NHS, the fraction of CERs $\leq \mathrm{C} 42$ was increased and the fraction of CERs $\geq \mathrm{C} 44$ was reduced in HSEs in relative amounts. Furthermore, the CERs EO chain length distribution was generally similar in NHS and HSEs in relative and absolute values, except for FTMs as these contain a higher level of CERs EO C70 and C72 per mg SC. Then, the CERs total $_{\text {subclass profiles were }}$ determined and compared in HSEs generated at varying $\mathrm{RH}$ levels for relative (Fig. 2f) and for absolute amounts (Supplementary Fig. S4). No differences were observed between high and low RH in both comparisons. As compared to NHS, alterations in the subclass profile (i.e., subclasses NS, NP, AS, and EOS) were detected in both HSE types. Considering the absolute amounts, the CC-FTMs resembled NHS to a higher extent in subclass profile, although important deviations persisted. Composition assessment was continued by the determination of the level of unsaturation in the CER subclasses AS and NS. Due to the low abundance of unsaturation of these CERs in NHS, these levels were not further analysed [2]. The percentage of unsaturation (i.e., unsaturation index) was determined in CER subclasses NS and AS (Fig. 2g). At 60\% RH, a reduction in the unsaturation index was observed for subclass NS as compared to high RH in CC-FTM, whereas in FTMs, no changes were observed due to altered RH. The unsaturation index for these a

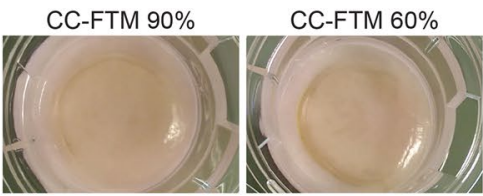

HE

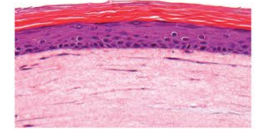

b

Epidermal thickness

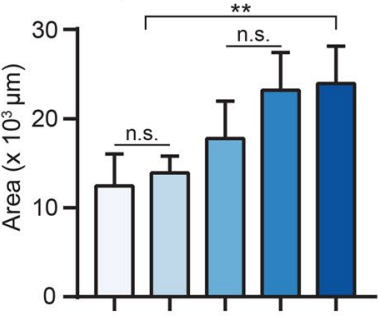

c
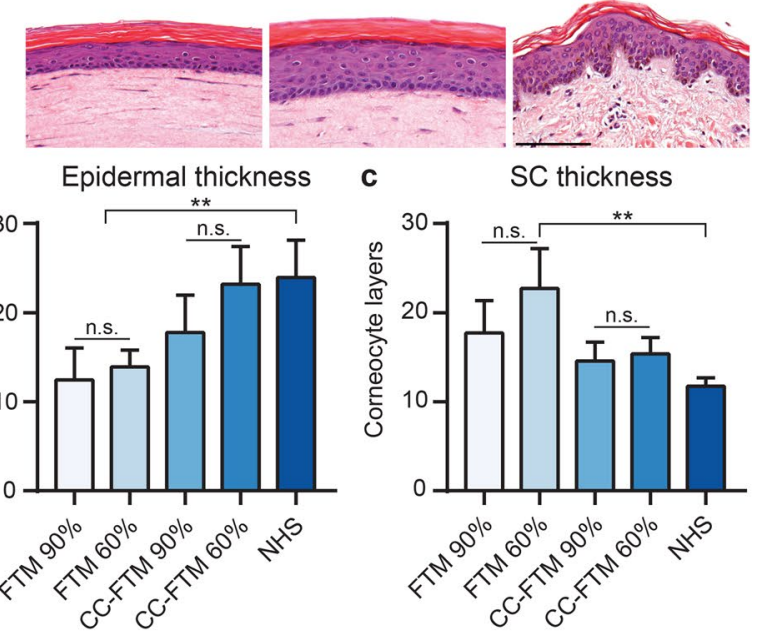

d
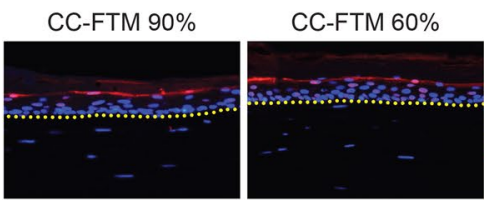

NHS

LOR

FLG
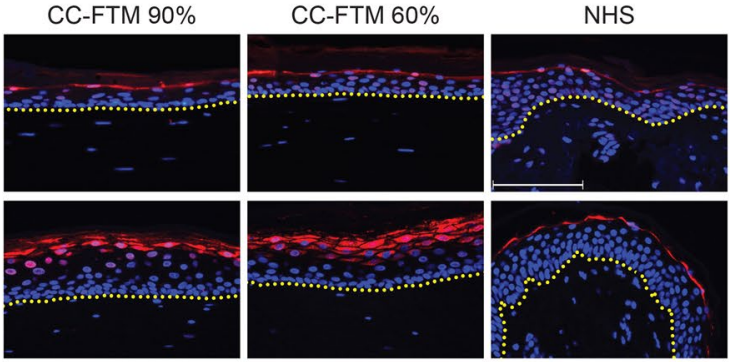

INV
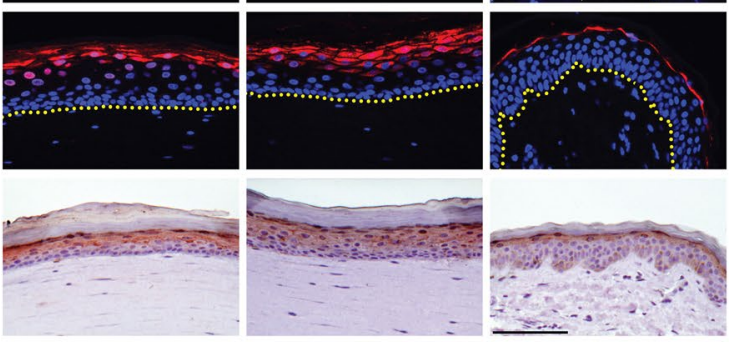

K10
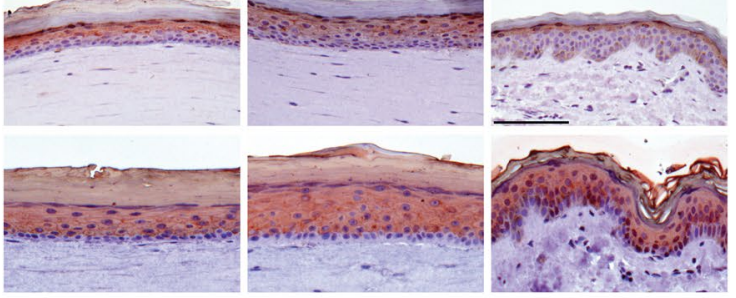

K16
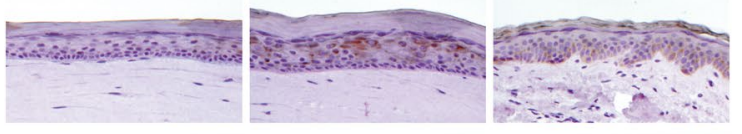

Ki67
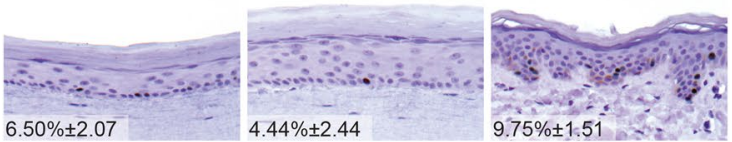

COL
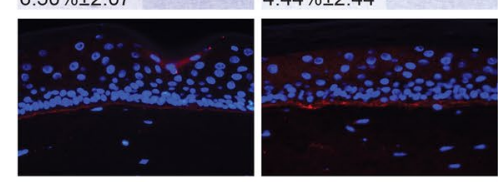

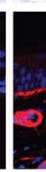

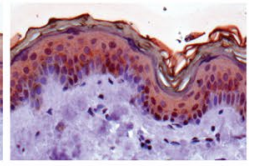

IM
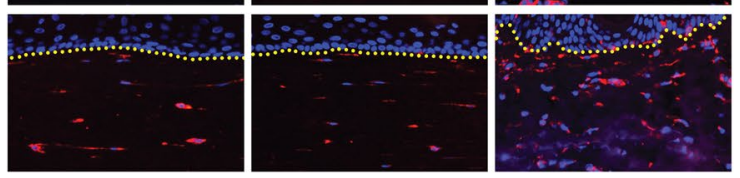
Fig. 2 Stratum corneum ceramide composition of HSEs generated at reduced relative humidity. a Representative ion maps of CC-FTMs generated at $90 \%$ and $60 \%$ RH and of NHS. Twelve CERs total $_{\text {subclasses }}$ are indicated by position in the ion map of NHS and are named according to Motta et al. [35]. Ion maps are shown as time after elution on the $x$-axis and mass in atomic mass unit (amu) on the $y$-axis. b Level of $\mathrm{CERs}_{\text {total }}$ ceramides in the SC per mg SC of indicated HSEs and of NHS. c Total amount of CERs in $1500 \mathrm{ng}$ lipid extract as detected by LC-MS. d Bar diagram plot of the mean carbon chain length of CERs. e Bar diagram plot showing the CERs EO mean carbon chain length. $\mathbf{f}$ CER and CER EO subclass profiles in relative amount. $\mathbf{g}$ Bar diagram plot of the unsaturation index in CER subclasses NS and AS of indicated HSEs. All data represent mean $+\mathrm{SD}, N=4$

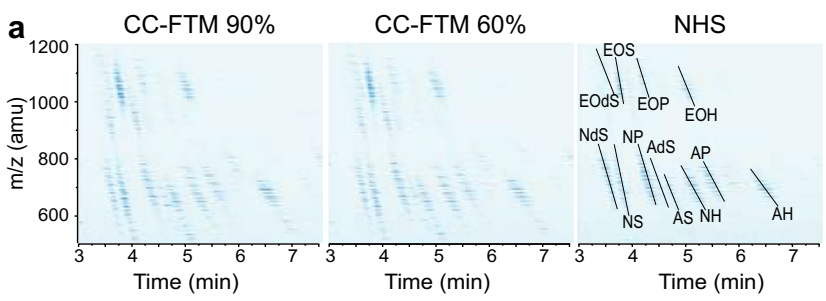

b

c Ceramides detected
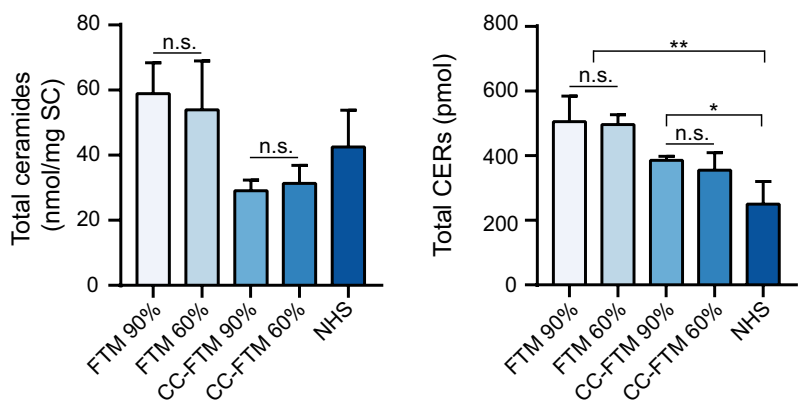

d

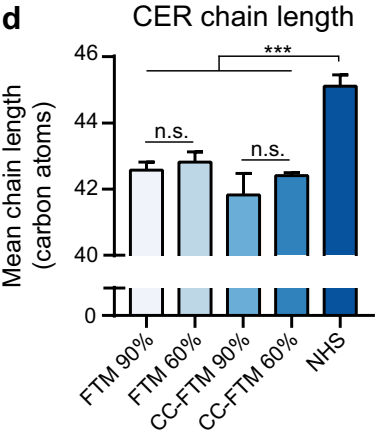

e CER EO chain length

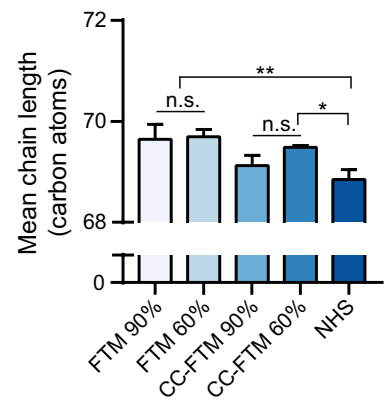

f CER subclass profile
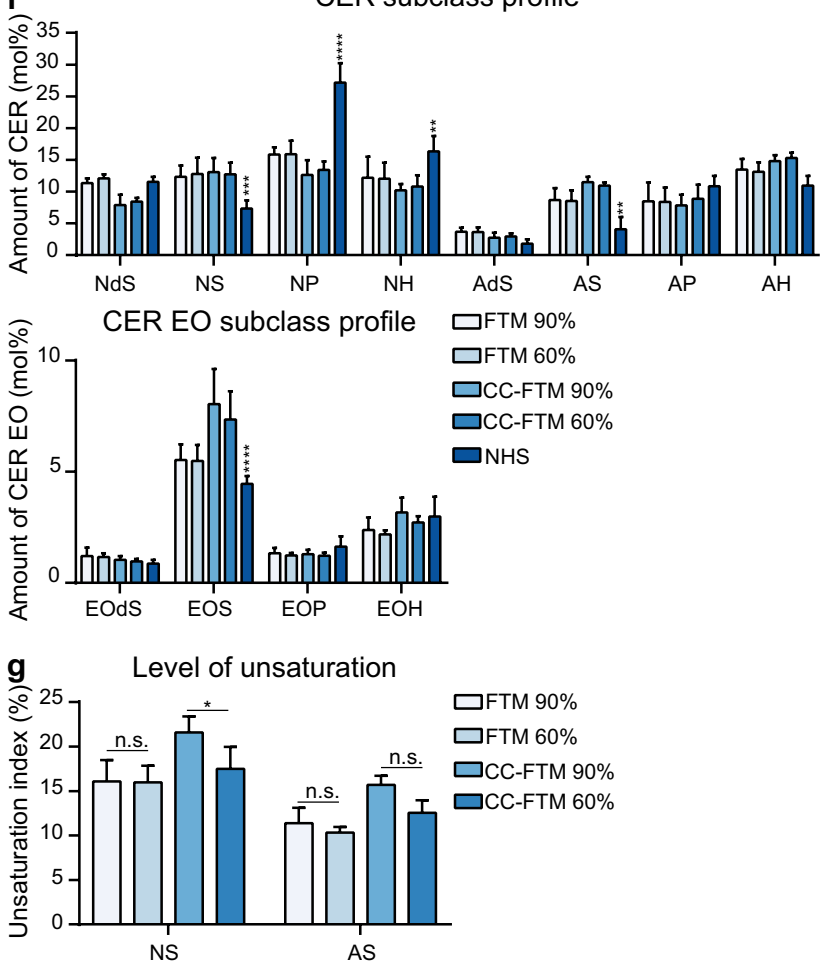
subclasses provides an indication on the change in level of unsaturation for the other CERs total $_{\text {subclasses. }}$ subs

\section{Glucosylceramide processing at varying RH levels}

We further studied the terminal ceramide processing which occurs at the SG-SC interface. The conversion of precursor ceramides into ceramides is mediated by the enzymes aSMASE and GBA (Fig. 3a). To fully comprehend the effect of RH on this process, we analysed enzyme protein expression, enzyme activity, and substrate-to-product ratio. In both HSE types, GBA expression was detected in the SG and aSMASE expression was highest in the SG, similar as in NHS (Fig. 3b). In CC-FTMs generated at $60 \%$ RH, GBA and aSMASE were present in more cell layers of the $\mathrm{SG}$, in line with the results of epidermal morphogenesis. Active GBA was detected in situ using the activity-based probe MDW941, a fluorescent suicide inhibitor. Active GBA molecules resided at the SG-SC interface and this distribution was similar at both RH levels and in NHS (Fig. 3c). The ratio between glucosylceramide (GlcCER) to ceramide was determined by LC-MS. The presence of the higher molecular weight GlcCERs for most of the CER subclasses was detected on the LC-MS ion map (Fig. 3d). We determined the relative GlcCER indexes for subclasses EOS and $\mathrm{EOH}$, which are exclusively processed by GBA [52]. In addition, this provides an indication for the GlcCER index

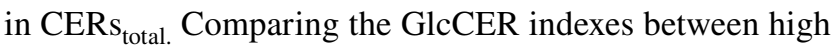
and low RH and to NHS, no significant differences were observed in this data set (Fig. 3e).

\section{Organization of the stratum corneum barrier lipids}

Analyses of the lipid organization provides additional information on the barrier formation at varying RH levels. The intercorneocyte lipid matrix contains repetitive lamellar stackings (Fig. 4a, b), which were studied by small-angle $\mathrm{X}$-ray diffraction. The diffraction profiles revealed the presence of the long periodicity phase (LPP) at $90 \%$ and $60 \%$ RH in both HSE types (Fig. 4c). The repeat distance of the LPP was longer in the CC-FTMs generated at reduced RH, whereas in FTMs, this remained equal. As compared to NHS, the LPP repeat distance is shorter and there is no SPP formed in both HSE types [5]. Within the lamellae, lipids are organized in either a very dense orthorhombic or in a dense hexagonal lateral packing (Fig. 4d). The lateral packing was determined in HSEs generated at high and low RH and appeared to be hexagonal in the CC-FTMs (Fig. 4e) as well as in the FTMs (data not shown), irrespective of RH level. In NHS, the lipids were predominantly ordered in the orthorhombic lateral packing.

\section{Discussion}

The objectives of this study were to determine the effects of RH on epidermal morphogenesis and on lipid barrier formation during in vitro development of HSEs. In addition, whether RH affects the conversion of GlcCER into CER was studied. This study shows that the reduction of RH mainly influenced the differentiation in the uppermost epidermal layers and that it resulted in epidermal activation, although variations between HSE types were observed. Strong alterations for neither SC ceramide composition nor lipid organization were observed. In addition, the conversion of GlcCER into CER at the SG-SC interface remained unaffected by variations in RH. By optimization and standardisation of the in vitro cell-culture conditions, more robust and clinically significant HSEs representing healthy and diseased skin will be generated. This makes HSEs a valuable tool in preclinical research.

Our data are in agreement with the diversity in adaptations after RH reduction in different HSE types. The FTMs data showed no effect on epidermal morphogenesis, which also has been observed before [6]. The CC-FTM data showed an enlarged SG at reduced $\mathrm{RH}$, in line with the previous results in epidermal models [7, 46]. More research is required to explain this diversity, although the dermis is likely to play a contributing factor in adaptation to reduced RH. Another morphogenetic adaptation to lower RH was the higher epidermal cell activation, which has been aggravated at levels below 60\% RH (J.A. Bouwstra, unpublished results). This could be due to an accelerated differentiation program and barrier formation as response to the lower external water level, inducing temporary but fierce signalling for barrier repair responses [20]. In addition, reduced RH levels affected the activation of dermal fibroblasts, which could contribute to more epidermal activation [56, 57]. As an activated epidermis is shown to undermine the lipid barrier formation in vitro and in vivo $[9,41]$, lower in vitro $\mathrm{RH}$ levels are not recommended.

The variance in lipid composition between high and low RH was small, leading to an unaltered SC lipid organization in HSEs. The differences in lipid composition between HSEs and NHS are related to the differences in lipid organization, which is in line with the previous reports $[48,50]$. Although the contribution of each lipid molecule to the matrix organization is not fully elucidated, it is known that major contributors to a reduced repeat distance of the LPP are the disbalanced CER subclass profile in combination with the reduced MCL of CERs [24]. The lack of formation of SPP is ascribed to the overabundance of CER EO subclasses [3, 11]. Moreover, the formation of a hexagonal lateral packing in HSEs is associated with 
Fig. 3 Precursor ceramide conversion in HSEs generated at reduced relative humidity. a Illustration of terminal ceramide processing mediated by $\beta$-glucocerebrosidase-1 (GBA) and acid sphingomyelinase (aSMASE). b Protein expression of GBA and aSMASE in HSEs and NHS. c Activitybased probe (ABP) fluorescence signal of MDW941, which solely binds to the active GBA enzyme. Protein expression and ABP signal are shown in red and nuclei are shown in blue, and yellow dotted line indicates dermal-epidermal junction. Scale bar indicates $100 \mu \mathrm{m}$. d Ion maps of the GlcCERs in CC-FTMs and NHS. e Relative values of the glucosylceramide index of CER EO subclasses EOS and EOH. Data represent mean $+\mathrm{SD}, N=4$

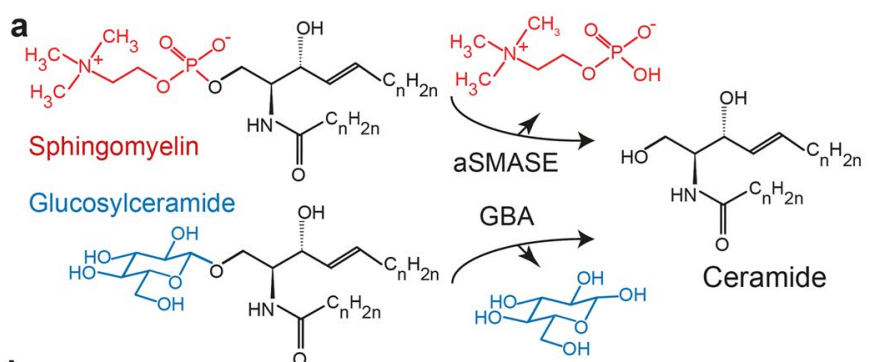

b

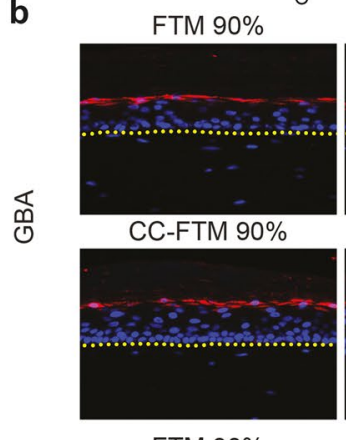

FTM $60 \%$

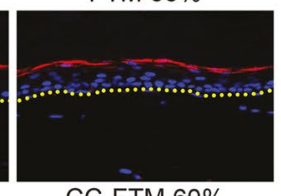

CC-FTM $60 \%$
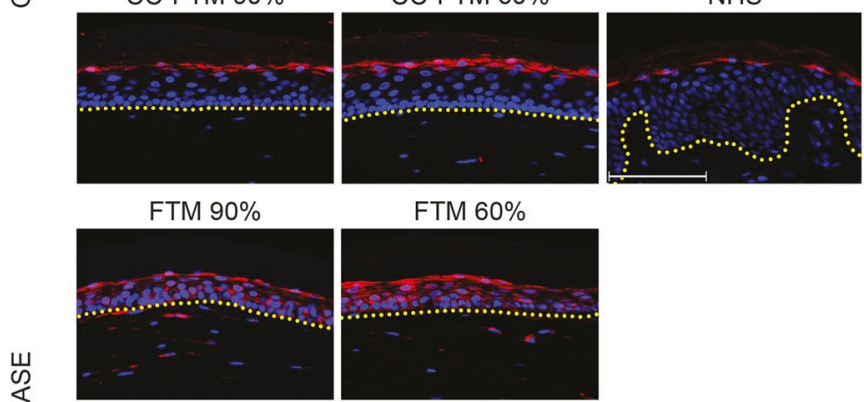

FTM $60 \%$

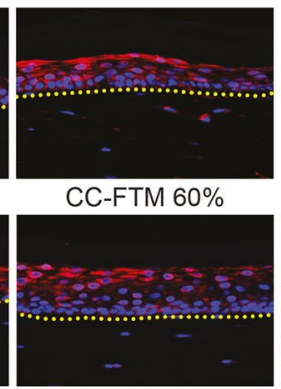

CC-FTM $60 \%$
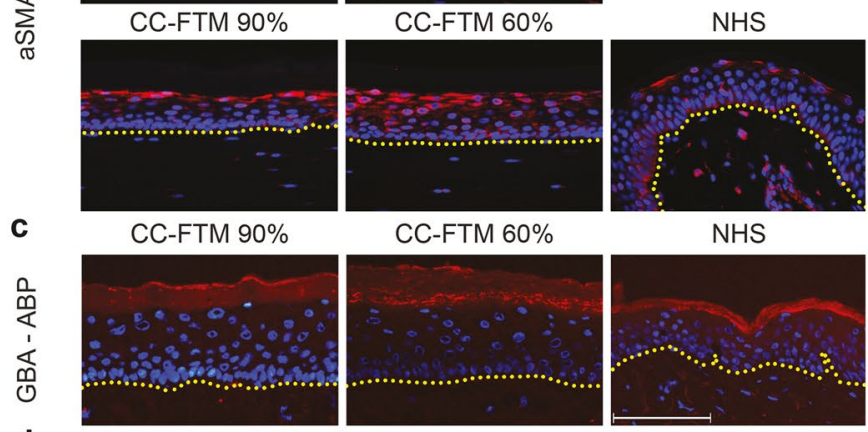

NHS

c

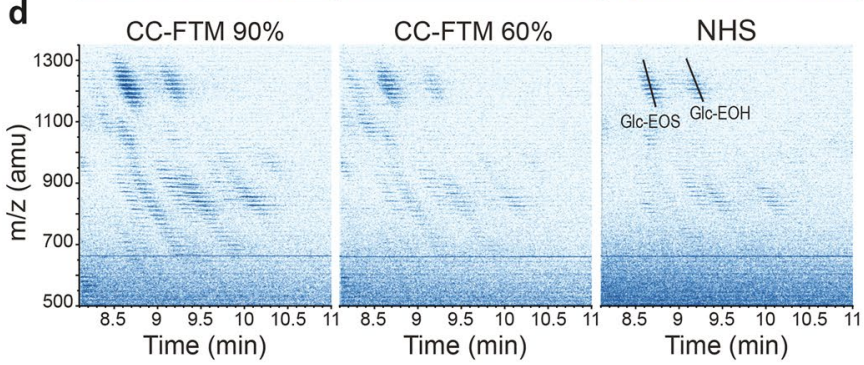

e

Glucosylceramide index

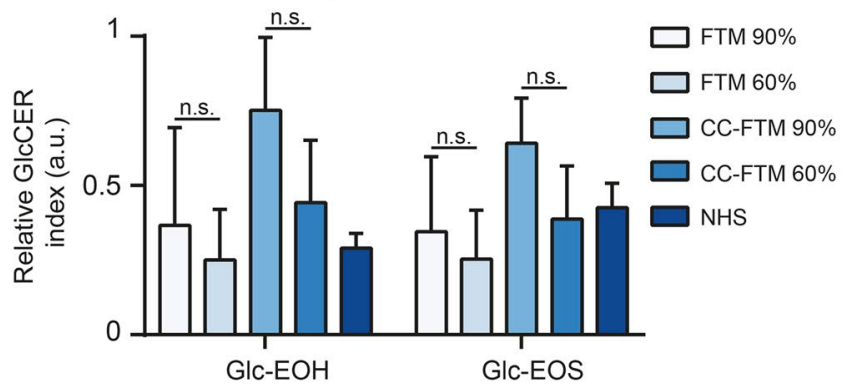




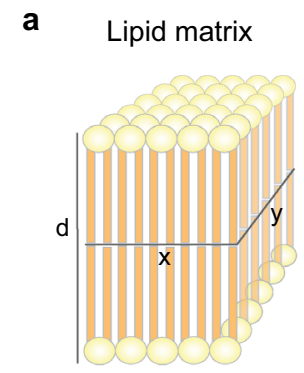

b

Lamellar organization
LPP

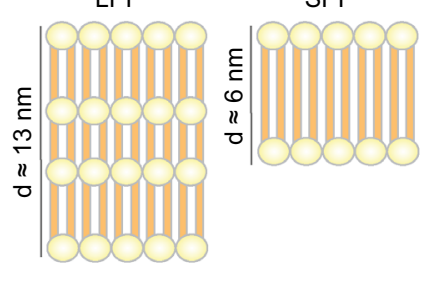

C

Diffraction profiles
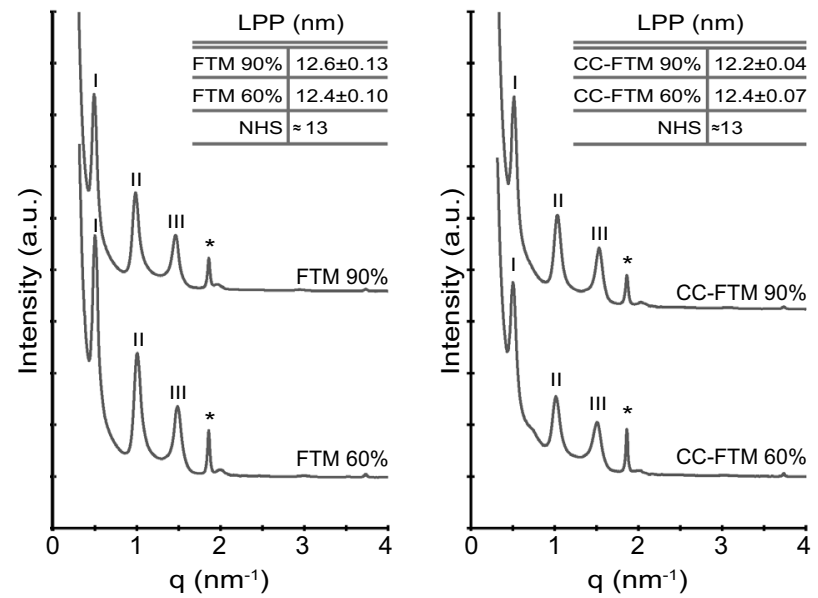

Fig. 4 Lipid organization in HSEs generated at reduced relative humidity. a Schematic overview of the 3D lipid matrix in the SC. Lipids are shown with head group region in yellow and hydrocarbon chain region in orange. b Illustration of the lamellar phases. The long periodicity phase (LPP) has an approximate repetition distance $(d)$ of $13 \mathrm{~nm}$ and the short periodicity phase (SPP) has an approximate $\mathrm{d}$ of $6 \mathrm{~nm}$ [5]. c Representative X-ray diffraction profiles. Peaks attributed to the LPP are indicated by Roman numbers. The peak of crystalline cholesterol phase is indicated by the asterisk symbol (*). Inset shows

the increased presence of monounsaturated CERs, and the increased presence of CERs with a shorter chain length $[24,50]$. These aspects remain important targets for future studies to better mimic the barrier formation of NHS in HSEs.

Novel insights on the effect of RH on the conversion of GlcCER into CERs were obtained. Earlier findings in dry $(50 \% \mathrm{RH}) \mathrm{HSEs}$ revealed an upregulated expression of glucosylceramide synthase, indicating an upregulation of total CER synthesis [46], whereas in submerged (>100\% RH) HSEs, an accumulation of GlcCER in the lipid matrix was observed [49]. Our results show similarity in GlcCER indexes, absolute ceramide content, GBA enzyme expression, and GBA activity at high and low RH levels. These complementary findings indicate that ceramide biosynthesis is not affected by reduced RH levels. Although in submerged HSEs there is an accumulation of GlcCERs [49], mediated by increased synthesis or reduced conversion

\section{d}
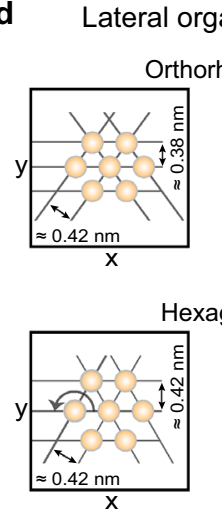

Hexagonal
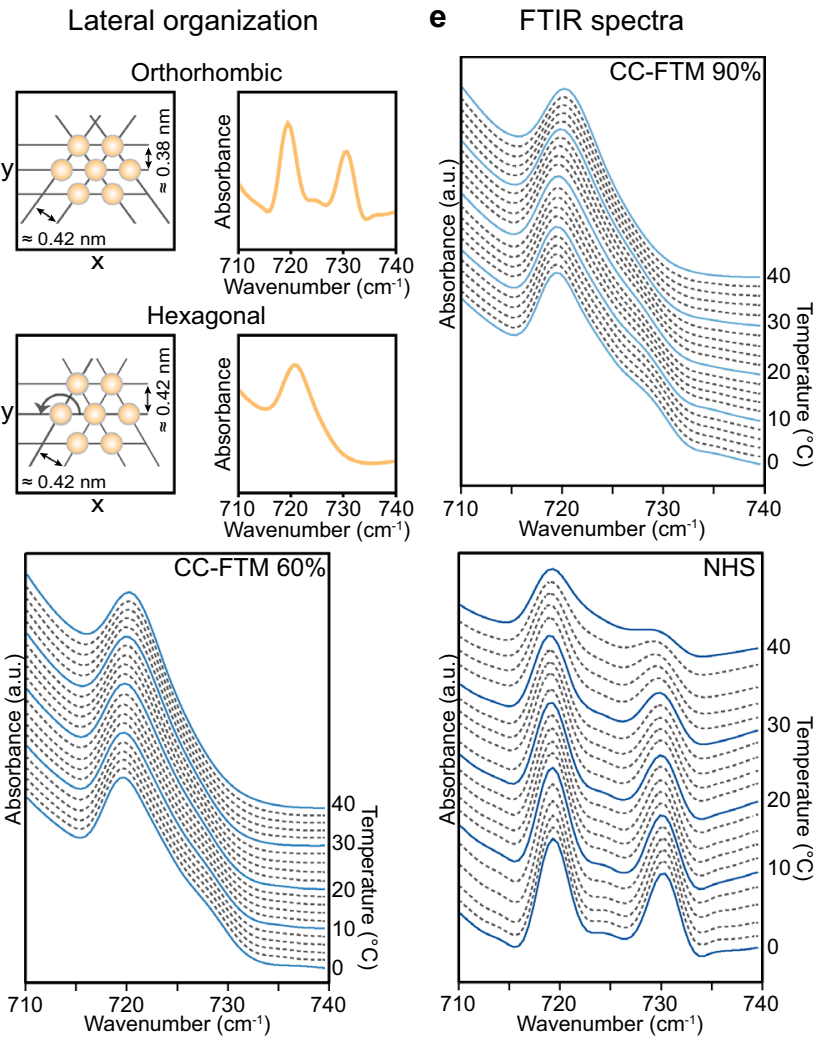

the repeat distances of the LPP as mean $\pm \mathrm{SD}, N=4$, whereas for NHS it was derived from Bouwstra et al. [5]. d Schematic overview of lateral lipid organization. Hydrocarbon chains can adopt a very dense orthorhombic or a dense hexagonal packing. This is indicated by a double or single peak, respectively, in the methylene rocking vibration region after measurement by FTIR. e Representative FTIR spectra of the methylene rocking vibrational mode of lipids in CCFTMs and NHS

after air-exposure, the terminal ceramide processing is not impeded by high in vitro RH levels.

The expected epidermal hyperplasia in FTMs was not observed in this study [31, 48], most probably caused by primary donor effects or increased rate of medium refreshments, but will not impair our conclusions [30, 31]. Furthermore, due to a high technical complexity in the data analysis, in this study, no unsaturated CERs EO with a linoleic chain (contributing 17-19\% to FTM and NHS; Helder et al., in preparation) and saturated CERs EO with an oleic chain (contributing 23\% to FTM; Helder et al., in preparation) were quantified. This leads to the underestimation of the abundance of CERs EO. Although FTMs and CC-FTMs were generated with different primary cell donors limiting a direct comparison between both, it appears reasonable that the morphogenesis and barrier formation in CC-FTMs better resemble that of NHS in line with the previous findings [30]. An overall limitation of both HSE types is the lack 
sweat and sebum production, of which the salt, glycerol, and lactate are known to affect the hydration status and barrier functionality of the SC $[6,17,22,27]$. These factors should also be considered in future studies regarding the interplay between RH and HSEs.

This study revealed that the reduction of external relative humidity levels during in vitro development of human skin equivalents does not lead to strong alterations in the epidermal lipid barrier formation. This study contributes with novel insights to a better understanding of the influence of cell-culture conditions on the lipid barrier formation in HSEs.

Acknowledgements The authors would like to thank Jeroen van Smeden for the support during the activity-based probe analyses. We also acknowledge Samira Absalah for the assistance during preparation and running of the lipidomic analyses. The authors are grateful for the support during the small-angle X-ray diffraction measurements by the personnel of the DUBBLE beam line (BM26) at the ESRF. The company Evonik (Essen, Germany) is thanked for the provision of ceramides. The work was funded by Grant no. 13151 of the Dutch Technology Foundation STW, which is part of the Netherlands Organisation for Scientific Research (NWO), and which is partly funded by the Ministry of Economic Affairs.

Author contributions AM, JAB, and AEG designed the research study. $\mathrm{AM}, \mathrm{RvD}$, and GG performed the research. AM and WB performed the data analysis. AM drafted the manuscript, which was commented on by HSO, JMFGA, JAB, and AEG. All authors approved the submitted manuscript.

\section{Compliance with ethical standards}

Conflict of interest The authors have declared no conflicting interests.

Open Access This article is distributed under the terms of the Creative Commons Attribution 4.0 International License (http://creativeco mmons.org/licenses/by/4.0/), which permits unrestricted use, distribution, and reproduction in any medium, provided you give appropriate credit to the original author(s) and the source, provide a link to the Creative Commons license, and indicate if changes were made.

\section{References}

1. Blair C (1968) Morphology and thickness of the human stratum corneum. Br J Dermatol 80:430-436. https://doi. org/10.1111/j.1365-2133.1968.tb11978.x

2. Boiten W, Absalah S, Vreeken R, Bouwstra J, van Smeden J (2016) Quantitative analysis of ceramides using a novel lipidomics approach with three dimensional response modelling. Biochim Biophys Acta BBA Mol Cell Biol Lipids 1861:1652-1661. https ://doi.org/10.1016/j.bbalip.2016.07.004

3. Boncheva M (2014) The physical chemistry of the stratum corneum lipids. Int J Cosmet Sci 36:505-515. https://doi.org/10.1111/ ics. 12162

4. Bouwstra JA, de Graaff A, Gooris GS, Nijsse J, Wiechers JW, van Aelst AC (2003) Water distribution and related morphology in human stratum corneum at different hydration levels. J Investig Dermatol 120:750-758. https://doi.org/10.104 6/j.1523-1747.2003.12128.x

5. Bouwstra JA, Gooris GS, van der Spek JA, Bras W (1991) Structural investigations of human stratum corneum by small-angle X-ray scattering. J Investig Dermatol 97:1005-1012. https://doi. org/10.1111/1523-1747.ep12492217

6. Bouwstra JA, Groenink HWW, Kempenaar JA, Romeijn SG, Ponec M (2008) Water distribution and natural moisturizer factor content in human skin equivalents are regulated by environmental relative humidity. J Investig Dermatol 128:378-388. https://doi.org/10.1038/sj.jid.5700994

7. Cau L, Pendaries V, Lhuillier E, Thompson PR, Serre G, Takahara H, Méchin M-C, Simon M (2017) Lowering relative humidity level increases epidermal protein deimination and drives human filaggrin breakdown. J Dermatol Sci 86:106-113. https://doi.org/10.1016/j.jdermsci.2017.02.280

8. Choe C, Schleusener J, Lademann J, Darvin ME (2017) Keratin-water-NMF interaction as a three layer model in the human stratum corneum using in vivo confocal Raman microscopy. Sci Rep 7:15900. https://doi.org/10.1038/s41598-017-16202-x

9. Choi MJ, Maibach HI (2005) Role of ceramides in barrier function of healthy and diseased skin. Am J Clin Dermatol 6:215223. https://doi.org/10.2165/00128071-200506040-00002

10. Dai A (2006) Recent climatology, variability, and trends in global surface humidity. J Clim 19:3589-3606. https://doi. org/10.1175/jcli3816.1

11. de Jager MW, Gooris GS, Dolbnya IP, Bras W, Ponec M, Bouwstra JA (2004) Novel lipid mixtures based on synthetic ceramides reproduce the unique stratum corneum lipid organization. J Lipid Res 45:923-932. https://doi.org/10.1194/jlr.M3004 84-JLR200

12. El Ghalbzouri A, Commandeur S, Rietveld MH, Mulder AA, Willemze R (2009) Replacement of animal-derived collagen matrix by human fibroblast-derived dermal matrix for human skin equivalent products. Biomaterials 30:71-78. https://doi.org/10.1016/j. biomaterials.2008.09.002

13. El Ghalbzouri A, Siamari R, Willemze R, Ponec M (2008) Leiden reconstructed human epidermal model as a tool for the evaluation of the skin corrosion and irritation potential according to the ECVAM guidelines. Toxicol In Vitro 22:1311-1320. https://doi. org/10.1016/j.tiv.2008.03.012

14. Elias PM (2012) Structure and function of the stratum corneum extracellular matrix. J Investig Dermatol 132:2131-2133. https:// doi.org/10.1038/jid.2012.246

15. Engebretsen K, Johansen J, Kezic S, Linneberg A, Thyssen J (2016) The effect of environmental humidity and temperature on skin barrier function and dermatitis. J Eur Acad Dermatol Venereol 30:223-249. https://doi.org/10.1111/jdv.13301

16. Fluhr JW, Elias PM (2002) Stratum corneum pH: formation and function of the 'acid mantle'. Exogen Dermatol 1:163-175. https ://doi.org/10.1159/000066140

17. Fluhr JW, Mao-Qiang M, Brown BE, Wertz PW, Crumrine D, Sundberg JP, Feingold KR, Elias PM (2003) Glycerol regulates stratum corneum hydration in sebaceous gland deficient (asebia) mice. J Investig Dermatol 120:728-737. https://doi.org/10.104 6/j.1523-1747.2003.12134.x

18. Goad N, Gawkrodger D (2016) Ambient humidity and the skin: the impact of air humidity in healthy and diseased states. J Eur Acad Dermatol Venereol 30:1285-1294. https://doi.org/10.1111/ jdv. 13707

19. Groen D, Poole DS, Gooris GS, Bouwstra JA (2011) Is an orthorhombic lateral packing and a proper lamellar organization important for the skin barrier function? Biochim Biophys Acta BBA Biomembr 1808:1529-1537. https://doi.org/10.1016/j. bbamem.2010.10.015 
20. Grubauer G, Elias PM, Feingold KR (1989) Transepidermal water loss: the signal for recovery of barrier structure and function. $\mathrm{J}$ Lipid Res 30:323-333

21. Haisma EM, Rietveld MH, de Breij A, van Dissel JT, El Ghalbzouri A, Nibbering PH (2013) Inflammatory and antimicrobial responses to methicillin-resistant Staphylococcus aureus in an in vitro wound infection model. PLoS ONE 8:e82800. https:// doi.org/10.1371/journal.pone.0082800

22. Hara M, Verkman A (2003) Glycerol replacement corrects defective skin hydration, elasticity, and barrier function in aquaporin-3-deficient mice. Proc Natl Acad Sci 100:7360-7365. https://doi.org/10.1073/pnas.1230416100

23. Hatano Y, Terashi H, Arakawa S, Katagiri K (2005) Interleukin-4 suppresses the enhancement of ceramide synthesis and cutaneous permeability barrier functions induced by tumor necrosis factor- $\alpha$ and interferon- $\gamma$ in human epidermis. J Investig Dermatol 124:786-792. https://doi.org/10.1111/j.0022202X.2005.23651.x

24. Janssens M, van Smeden J, Gooris GS, Bras W, Portale G, Caspers PJ, Vreeken RJ, Hankemeier T, Kezic S, Wolterbeek R (2012) Increase in short-chain ceramides correlates with an altered lipid organization and decreased barrier function in atopic eczema patients. J Lipid Res. https://doi.org/10.1194/jlr.P030338

25. Kihara A (2016) Synthesis and degradation pathways, functions, and pathology of ceramides and epidermal acylceramides. Prog Lipid Res 63:50-69. https://doi.org/10.1016/j.plipres.2016.04.001

26. Kuntsche J, Bunjes H, Fahr A, Pappinen S, Rönkkö S, Suhonen M, Urtti A (2008) Interaction of lipid nanoparticles with human epidermis and an organotypic cell culture model. Int J Pharm 354:180-195. https://doi.org/10.1016/j.ijpharm.2007.08.028

27. Lee A-RC, Moon HK (2007) Gravimetric analysis and differential scanning calorimetric studies on glycerin-induced skin hydration. Arch Pharmacal Res 30:1489-1495. https://doi.org/10.1007/bf029 77376

28. Mak VHW, Cumpstone MB, Kennedy AH, Harmon CS, Guy RH, Potts RO (1991) Barrier function of human keratinocyte cultures grown at the air-liquid interface. J Investig Dermatol 96:323-327. https://doi.org/10.1111/1523-1747.ep12465212

29. Mieremet A, García AV, Boiten W, van Dijk R, Gooris G, Bouwstra JA, El Ghalbzouri A (2019) Human skin equivalents cultured under hypoxia display enhanced epidermal morphogenesis and lipid barrier formation. Sci Rep 9:7811

30. Mieremet A, Rietveld M, Absalah S, van Smeden J, Bouwstra JA, El Ghalbzouri A (2017) Improved epidermal barrier formation in human skin models by chitosan modulated dermal matrices. PLoS ONE 12:e0174478. https://doi.org/10.1371/journal.pone.0174478

31. Mieremet A, Rietveld M, van Dijk R, Bouwstra JA, El Ghalbzouri A (2017) Recapitulation of native dermal tissue in a full-thickness human skin model using human collagens. Tissue Eng Part A. https://doi.org/10.1089/ten.tea.2017.0326

32. Mieremet A, van Dijk R, Gooris G, Bouwstra JA, El Ghalbzouri A (2019) Shedding light on the effects of 1, 25-dihydroxyvitamin D3 on epidermal lipid barrier formation in three-dimensional human skin equivalents. J Steroid Biochem Mol Biol 189:19-27

33. Mojumdar EH, Helder RW, Gooris GS, Bouwstra JA (2014) Monounsaturated fatty acids reduce the barrier of stratum corneum lipid membranes by enhancing the formation of a hexagonal lateral packing. Langmuir 30:6534-6543. https://doi.org/10.1021/ la500972w

34. Mojumdar EH, Pham QD, Topgaard D, Sparr E (2017) Skin hydration: interplay between molecular dynamics, structure and water uptake in the stratum corneum. Sci Rep 7:15712. https:// doi.org/10.1038/s41598-017-15921-5

35. Motta S, Monti M, Sesana S, Caputo R, Carelli S, Ghidoni R (1993) Ceramide composition of the psoriatic scale.
Biochim Biophys Acta Mol Basis Dis 1182:147-151. https://doi. org/10.1016/0925-4439(93)90135-N

36. Niehues H, Bouwstra JA, Waheb El Ghalbzouri A, Brandner JM, Zeeuwen PL, van den Bogaard EH (2018) 3D skin models for 3R research: the potential of 3D reconstructed skin models to study skin barrier function. Exp Dermatol. https://doi.org/10.1111/ exd.13531

37. Nolte CJ, Oleson MA, Bilbo PR, Parenteau NL (1993) Development of a stratum corneum and barrier function in an organotypic skin culture. Arch Dermatol Res 285:466-474. https://doi. org/10.1007/BF00376819

38. Ponec M, Boelsma E, Weerheim A, Mulder A, Bouwstra J, Mommaas M (2000) Lipid and ultrastructural characterization of reconstructed skin models. Int J Pharm 203:211-225. https://doi. org/10.1016/S0378-5173(00)00459-2

39. Ponec M, Weerheim A, Kempenaar J, Mulder A, Gooris GS, Bouwstra J, Mommaas AM (1997) The formation of competent barrier lipids in reconstructed human epidermis requires the presence of vitamin C. J Investig Dermatol 109:348-355. https://doi. org/10.1111/1523-1747.ep12336024

40. Proksch E, Brandner JM, Jensen JM (2008) The skin: an indispensable barrier. Exp Dermatol 17:1063-1072. https://doi.org/1 0.1111/j.1600-0625.2008.00786.x

41. Proksch E, Fölster-Holst R, Jensen J-M (2006) Skin barrier function, epidermal proliferation and differentiation in eczema. J Dermatol Sci 43:159-169. https://doi.org/10.1016/j.jderm sci.2006.06.003

42. Rawlings AV, Harding CR (2004) Moisturization and skin barrier function. Dermatol Ther 17:43-48. https://doi.org/10.1111/ j.1396-0296.2004.04S1005.x

43. Rissmann R, Oudshoorn MH, Hennink WE, Ponec M, Bouwstra JA (2009) Skin barrier disruption by acetone: observations in a hairless mouse skin model. Arch Dermatol Res 301:609-613. https://doi.org/10.1007/s00403-009-0946-6

44. Sakai S, Endo Y, Ozawa N, Sugawara T, Kusaka A, Sayo T, Inoue S, Tagami H (2003) Characteristics of the epidermis and stratum corneum of hairless mice with experimentally induced diabetes mellitus. J Investig Dermatol 120:79-85. https://doi.org/10.104 6/j.1523-1747.2003.12006.x

45. Sato J, Denda M, Chang S, Elias PM, Feingold KR (2002) Abrupt decreases in environmental humidity induce abnormalities in permeability barrier homeostasis. J Investig Dermatol 119:900-904. https://doi.org/10.1046/j.1523-1747.2002.00589.x

46. Sun R, Celli A, Crumrine D, Hupe M, Adame LC, Pennypacker SD, Park K, Uchida Y, Feingold KR, Elias PM (2014) Lowered humidity produces human epidermal equivalents with enhanced barrier properties. Tissue Eng Part C Methods 21:15-22. https:// doi.org/10.1089/ten.tec.2014.0065

47. Supp AP, Wickett RR, Swope VB, Harriger MD, Hoath SB, Boyce ST (1999) Incubation of cultured skin substitutes in reduced humidity promotes cornification in vitro and stable engraftment in athymic mice. Wound Repair Regener 7:226-237. https://doi. org/10.1046/j.1524-475X.1999.00226.X

48. Thakoersing VS, Gooris GS, Mulder A, Rietveld M, El Ghalbzouri A, Bouwstra JA (2011) Unraveling barrier properties of three different in-house human skin equivalents. Tissue Eng Part C Methods 18:1-11. https://doi.org/10.1089/ten.tec.2011.0175

49. Thakoersing VS, Ponec M, Bouwstra JA (2010) Generation of human skin equivalents under submerged conditions-mimicking the in utero environment. Tissue Eng Part A 16:1433-1441. https ://doi.org/10.1089/ten.TEA.2009.0358

50. Thakoersing VS, van Smeden J, Mulder AA, Vreeken RJ, El Ghalbzouri A, Bouwstra JA (2013) Increased presence of monounsaturated fatty acids in the stratum corneum of human skin equivalents. J Investig Dermatol 133:59-67. https://doi.org/10.1038/ jid.2012.262 
51. van Drongelen V, Danso MO, Mulder A, Mieremet A, van Smeden J, Bouwstra JA, El Ghalbzouri A (2014) Barrier properties of an N/TERT-based human skin equivalent. Tissue Eng Part A 20:3041-3049. https://doi.org/10.1089/ten.tea.2014.0011

52. van Smeden J, Dijkhoff IM, Helder RW, Al-Khakany H, Boer DE, Schreuder A, Kallemeijn WW, Absalah S, Overkleeft HS, Aerts JM (2017) In situ visualization of glucocerebrosidase in human skin tissue: zymography versus activity-based probe labeling. J Lipid Res 58:2299-2309. https://doi.org/10.1194/j1r.M079376

53. van Smeden J, Janssens M, Gooris GS, Bouwstra JA (2014) The important role of stratum corneum lipids for the cutaneous barrier function. Biochim Biophys Acta Mol Cell Biol Lipids 1841:295313. https://doi.org/10.1016/j.bbalip.2013.11.006

54. Vávrová K, Kováčik A, Opálka L (2017) Ceramides in the skin barrier. Eur Pharm J 64:28-35. https://doi.org/10.1515/afpuc $-2017-0004$

55. Vyumvuhore R, Tfayli A, Duplan H, Delalleau A, Manfait M, Baillet-Guffroy A (2013) Effects of atmospheric relative humidity on Stratum Corneum structure at the molecular level: ex vivo Raman spectroscopy analysis. Analyst 138:4103-4111. https:// doi.org/10.1039/c3an00716b

56. Zhao J, Yu J, Xu Y, Chen L, Zhou F, Zhai Q, Wu J, Shu B, Qi S (2018) Epidermal HMGB1 activates dermal fibroblasts and causes hypertrophic scar formation in reduced hydration. J Investig Dermatol. https://doi.org/10.1016/j.jid.2018.04.036

57. Zhao J, Zhong A, Friedrich EE, Jia S, Xie P, Galiano RD, Mustoe TA, Hong SJ (2017) S100A12 induced in the epidermis by reduced hydration activates dermal fibroblasts and causes dermal fibrosis. J Investig Dermatol 137:650-659. https://doi. org/10.1016/j.jid.2016.10.040

Publisher's Note Springer Nature remains neutral with regard to jurisdictional claims in published maps and institutional affiliations. 